\title{
Beyond transplant: Roles of atrial septostomy and Potts shunt in pediatric pulmonary hypertension
}

\author{
Ronald Grady ${ }^{1}$ \\ ${ }^{1}$ Washington University in Saint Louis School of Medicine
}

June 4, 2020

\begin{abstract}
Despite significant successes in treating children with pulmonary hypertension, many still die from their disease. Death most often results from right ventricular failure. Bilateral lung transplantation has historically been the ultimate palliation for children who develop heart failure despite maximal medical therapy. Creating an atrial level shunt has been advocated as a pre-transplant intervention to help reduce symptoms and perhaps serve as a bridge to transplant. More recently, the utilization of a pulmonary-to-systemic anastomosis, i.e., Potts shunt, has demonstrated efficacy in ameliorating symptoms in children with severe, progressive disease. This improvement is often coincident with a reduction in expensive and onerous pulmonary hypertension specific medications. Improvement has been sustained over years in some children, delaying the need for transplant consideration. Research is ongoing to determine more specific risk/benefits of using the Potts shunt in children with pulmonary hypertension both in the short term and long.
\end{abstract}

\section{Keywords}

pediatric pulmonary hypertension, atrial septostomy, Potts shunt

\section{Running Head}

Potts shunt and pediatric pulmonary hypertension

\section{Introduction}

The treatment of children with pulmonary hypertension $(\mathrm{PH})$ has evolved dramatically over the last 30 years. Early data demonstrated a median survival of less than one year whereas current estimates now predict 5-year survival rates of at least $80 \%$. ${ }^{1,2,3}$ This improvement likely reflects the development of PHspecific drugs and an increased awareness and understanding of PH pathology along with the development of specialized centers and personnel to treat it. Nonetheless, despite these advances, children still die from their disease. Children with severe PH who fail aggressive medical management have limited options. Historically, lung transplantation has been the palliative procedure most often considered in these critically compromised children. However, survival with pediatric lung transplantation remains relatively limited with current median survival of $\sim 7$ years in children transplanted for idiopathic $\mathrm{PH}$ and even lower survival for children with non-idiopathic $\mathrm{PH}$, a median of ${ }^{\sim} 3$ years. ${ }^{4,5}$ In addition, lung transplantation involves expensive, highly technical and specialized care that only a relatively small number of centers around the world can afford to offer. Consequently, care providers have looked for other ways in which to treat these medically refractory children. Ultimately, most children with severe $\mathrm{PH}$ die because of right ventricular failure. Thus, a number of interventions have developed over the years as a means to ameliorate right ventricular dysfunction short of transplantation. Physiologically these procedures fall into two categories: 1) creating a pre-tricuspid valve shunt, essentially making an atrial septal defect, or 2) creating a post-tricuspid valve shunt, typically a pulmonary-to-systemic anastomosis at the level of the great vessels, e.g., a Potts shunt. We will discuss 
these options in more detail. Other interventions for supporting the failing right ventricle such as ECMO, paracorporeal lung assist devices or right ventricular assist devices are therapies that are utilized almost exclusively in the intensive care setting and will not be covered in this article.

\section{Atrial Septostomy and Pulmonary Hypertension}

Several observations in the 1980's suggested that adult patients with severe, symptomatic PH who also had a patent foramen ovale had a survival advantage over those who did not. ${ }^{6,7}$

Improvement in those with atrial defects was postulated to result from the shunt "decompressing" the failing right ventricle, allowing for right to left atrial flow, thereby improving left ventricular preload and thus augmenting cardiac output and oxygen delivery (albeit with overall reduced systemic oxygen saturations). A number of relatively small case series followed, primarily in adults, demonstrating that in the select patient creating an atrial shunt via atrial septostomy (AS) could improve symptoms and possibly serve as a bridge to transplant. ${ }^{8,9}$ Whether AS improves survival however is less clear due to the lack of control cases. Nonetheless, several studies have argued that when compared to historical controls or calculated survival, patients who underwent AS fared better. ${ }^{9}$ Studies that look specifically at AS in children are few. One retrospective study reviewed 20 children with a mean age of 8.4 years who underwent AS. ${ }^{10}$ All were World Health organization functional class (WHO-FC) III or IV, 13 suffered from recurrent syncope. There were no procedural deaths. Post-procedure, functional class improved significantly and syncope was abolished in 12 of the 13 children. With a mean follow-up of 2.1 years, clinical improvement was maintained in 18 of the 20 children. Notably however, despite clinical improvement post-AS, 9 of the children (45\%) required the addition of intravenous epoprostenol to their medical regimen. Overall, enough evidence exists to recommend including AS as part of the standard, pediatric PH treatment algorithm for symptomatic children on maximal medical therapy. ${ }^{11}$ The importance of patient selection and technique in regards to a successful outcome dictate that this relatively high risk procedure (mortality over $20 \%$ in some adult series) should be performed in centers with significant experience. ${ }^{9}$

The ability of a pre-tricuspid (atrial) shunt to provide symptomatic relief in a child with $\mathrm{PH}$ depends upon having right atrial pressures high enough to force blood to flow from the right atrium to the left. Assuming a competent tricuspid valve, right atrial pressure is directly reflective of right ventricular end diastolic pressure (RVEDP). Consequently, for an atrial shunt to benefit a PH patient, the RVEDP must be abnormally elevated to generate right-to-left atrial flow. This elevation of RVEDP can happen acutely, as with exercise, or as the result of progressive right ventricular failure with decreasing systolic function and concomitantly rising RVEDP. Thus, a child with $\mathrm{PH}$ who improves from an AS is often one whose right ventricular failure is relatively advanced, perhaps suggesting why children who have had an AS may still require additional PH medications and where survival outcomes may not be obviously improved. ${ }^{10,12,13}$ This relatively "end stage" benefit of an AS contrasts with the potential advantage that a post-tricuspid shunt has to intervene much earlier in a child's disease course.

\section{Potts Shunt and Pulmonary Hypertension}

Typically, creation of a post-tricuspid shunt involves an anastomosis between the descending aorta and the left pulmonary artery, a surgical technique first described in 1946 by Dr. Willis Potts. ${ }^{14}$ The original goal of his eponymous shunt was to provide pulmonary blood flow to a child with tetralogy of Fallot at a time when cardiopulmonary bypass was not available. The procedure fell out of favor once primary repair became standard. However, in 2004, a group from Hôpital Necker-Enfants Malades in Paris, France resurrected the Potts shunt to treat two boys suffering from severe, suprasystemic right ventricular systolic pressures. ${ }^{15}$ Their insightful theory postulated that by creating an unrestrictive pulmonary-to-systemic shunt, the afterload on the right ventricle would be reduced to systemic levels and thereby recapitulate the pathophysiology seen in patients with Eisenmenger's syndrome. By doing so, the researchers hoped to confer upon these children the relatively superior life expectancy purported for patients with Eisenmenger's syndrome. ${ }^{16,17}$ One immediate consequence of the shunt would be to send deoxygenated blood to the lower half of the body, while preserving the highest oxygenated blood for the upper half and the brain in particular. This contrasts with an AS that 
leads to global deoxygenation. The two children experienced significant improvement both clinically and hemodynamically with follow-up at 6 and 18 months respectively.

This initial success led the way for their publication of 24 consecutive children with PH treated with a Potts shunt in $2012 .{ }^{18}$ In this series, children's ages ranged from 1.5 to 17 years with the majority classified as severely compromised with WHO-FC IV. Nineteen of the children had a traditional shunt via a left thoracotomy and direct anastomosis of the pulmonary artery and the aorta. Five had transcatheter shunts through the stenting of a restrictive patent ductus arteriosus (PDA). There were 3 procedural deaths (12.5\%). Children that went home had a median follow-up of 2.1 years with 7 children having over 8 years followup post-procedure. There was rapid clinical and hemodynamic improvement with better WHO-FC (from a median FC IV to II), longer 6 minute walk distances, lower brain natriuretic peptide (BNP) levels and improved echocardiographic findings. Perhaps most remarkable, these improvements occurred while weaning $\mathrm{PH}$ medications. Indeed, 6 of the children that were on intravenous epoprostenol pre-operatively were weaned completely off post-shunt. This contrasts with studies of patients undergoing AS for palliation where medical regiments often increased post-procedure. ${ }^{10,13}$ Overall, this series demonstrated that a child with severe $\mathrm{PH}$, WHO-FC IV on intravenous prostacyclin therapy, could experience a sustained, prostacyclin-free, improved quality of life by means of a Potts shunt.

In 2016, a group from Washington University in St Louis, USA published a series of five consecutive children who had undergone a surgical Potts shunt for severe $\mathrm{PH}^{19}$ Their findings mirrored that of the Parisian group, demonstrating in 4 of the children, with less follow-up (mean 10 months), an improvement in functional class and BNP levels. Since that publication, the St Louis group has performed a total of 23 Potts shunts in children with a mean age of 10.3 years (range $0.3-20$ years) with diagnoses including idiopathic $\mathrm{PH}$, heritable $\mathrm{PH}, \mathrm{PH}$ associated with congenital heart disease and $\mathrm{PH}$ associated with lung disease (personal observation). Twenty had surgical shunts, either through 1) direct anastomosis, 2) the use of a short inter-arterial graft or 3) with a valved conduit placed via a sternotomy. Three children had catheter-based interventions, two with PDA stenting and one with reopening a previously device-closed duct. Four of the children died (17\%) within 30 days of the procedure. Three of these early mortalities were children who presented in extremis, requiring veno-arterial ECMO prior to their shunt. The fourth child died from progressive hypoxia due to interstitial lung disease. Nineteen of the children were discharged home with a mean follow-up of 2.5 years (range 0.5-6 years). Seventeen of these children showed improvement clinically with lower WHO-FC scores, lower BNP levels and a reduction in overall $\mathrm{PH}$ medications. There were four late deaths and two lung transplants post-shunt. Reasons for death or transplant included worsening right ventricular failure, acute infection and one due to accidental tracheostomy tube dislodgement.

The data from Paris and St Louis demonstrate, with a combined mean follow-up of 2-3 years, that a Potts shunt can provide symptomatic improvement in children with severe $\mathrm{PH}$. The question of longer-term benefits, however, remains open. The original idea behind utilizing a Potts shunt was to "convert" a child to Eisenmenger-like physiology and possibly confer the purported survival benefits seen with Eisenmenger patients. This conferral of benefit assumes that a child with PH due to an etiology distinct from Eisenmenger's syndrome would respond similarly to right ventricular decompression. In adult series, median survival with Eisenmenger syndrome has been estimated as long as 40-60 years, far better than that reported in children with PH. ${ }^{2,3,16,17}$ The survival rate for children with Eisenmenger's syndrome, however, may not be as robust as seen in adults, with one estimate of $77 \%$ survival at four years. ${ }^{20}$ Nonetheless, even this reduced survival projection would still compare favorably to that of children who typically present for Potts shunt consideration, specifically, those who are WHO FC IV despite PH specific therapy. These compromised children have a purported median survival of less than two years. ${ }^{21}$

To address survival benefits and other aspects regarding the use of a Potts shunt in treating children with $\mathrm{PH}$, an international registry with at least fourteen centers has begun to compile data. Even without registry results, however, there are relevant observations and discussions that can be made regarding the Potts shunt and PH. From the St. Louis experience, three children were sick enough prior to their shunt procedure to require veno-arterial ECMO (personal observation). All three had surgical shunts and all three died in 
house within 30 days of their procedure. These results would suggest that in the severely decompensated child, a Potts shunt might not be an effective option. In fact, if these three children were excluded, acute mortality in the St Louis series would drop from $17 \%$ to less than $5 \%$. Thus, as with most procedures, increased experience in patient selection can lead to improved surgical outcomes. Certainly, if a child's anatomy allows, dilating and stenting a restrictive PDA would be the route of choice for creating a shunt, avoiding the potential morbidity from surgery. Unfortunately, this option is seldom available. Several centers have reported on creating a transcatheter shunt through direct puncture from the aorta to the pulmonary artery with subsequent placement of a covered stent. ${ }^{22,23}$ Between the two series, a total of ten patients underwent this transcatheter procedure with four deaths, an early mortality more than double of that seen in the St Louis and Paris experience. This relatively high risk may preclude the transcatheter Potts shunt from gathering widespread acceptance.

Not all children with severe PH will benefit from a Potts shunt. In theory, because the shunt is an unrestrictive communication between the pulmonary and systemic circulations, it will only be effective in reducing right ventricular afterload in those whose right ventricular systolic pressures are higher than the left, i.e., suprasystemic. Documenting suprasystemic right ventricular pressures can be done with either echocardiography or cardiac catheterization. Relying on right ventricular ejection fraction alone, however, is not sufficient. In the St Louis series, one child had a calculated right ventricular ejection fraction by MRI of $16 \%$ yet the echocardiogram still demonstrated suprasystemic pressures. Post-procedure this child did well clinically (personal observation). While a Potts shunt can successfully lower suprasystemic right ventricular pressures, subsequent pressures will still remain at abnormally high systemic levels. Consequently, a shunt placed in a child whose right ventricle has lost the ability to generate systemic to suprasystemic pressures may prove ineffectual. As a case in point, one child, early on in the St Louis series, was diagnosed initially with suprasystemic right ventricular pressures. ${ }^{19}$ At the time of his shunt however, echocardiograms demonstrated at most systemic right-sided pressures. The child failed to improve post-procedure and eventually died three months later from progressive right ventricular failure. Thus, intervening with a Potts shunt should occur before the onset of frank right ventricular failure, i.e., falling, sub-systemic, right ventricular systolic pressures with rising RVEDP.

In theory, a post-tricuspid shunt like a Potts has the potential to intercede relatively early in a child's disease course, while systolic function is still preserved at suprasystemic levels and diastolic pressures are comparatively low. This contrasts with an AS which relies upon an elevated RVEDP to be effective, a situation more typical of end-stage disease. This potential advantage of a Potts shunt over AS, leads to the interesting question as to how early in a child's disease course does one consider a shunt. Current recommendations suggest considering a Potts shunt in a child with severe, symptomatic disease who has failed aggressive medical therapy, including prostacyclin infusion. ${ }^{11}$ However, does waiting for such a child to develop symptoms reduce a shunts potential benefits and increase its risks? Those answers are currently unclear. What is clear, however, is that managing a 24-hour prostacyclin infusion places a substantial burden upon a child and family and that a Potts shunt offers the hope of easing that onus. Thus, perhaps intervention should be considered in a child with severe PH who is still WHO FC I-II rather than III-IV. A technical development that might provide impetus for employing a Potts shunt earlier in a child's course is the use of a valved conduit to create the inter-arterial anastomosis. A shunt that allows for only right-to-left flow might benefit the child who at rest has systemic to even sub-systemic right ventricular pressures yet with exertion develops suprasystemic pressures with resulting symptoms. A recently published series demonstrates the feasibility of such an approach. ${ }^{24}$ Managing the inevitable development of valve incompetency and a conduit's limitations in a growing child would need to be considered.

For children with severe, progressive, medically recalcitrant $\mathrm{PH}$, bilateral lung transplantation remains the ultimate palliative option. Whether a Potts shunt can provide a similar survival rate as lung transplantation requires more data. A more immediate question, however, is whether having a Potts shunt precludes further consideration for lung transplantation. There is one reported case from the Paris series of a child who had a Potts shunt and subsequently underwent successful lung transplantation. ${ }^{25}$ This child initially did well post-shunt with clinical improvement and weaning from her intravenous epoprostenol. However, six years 
after her shunt, she developed severe, persistent hemoptysis and was listed for transplant. On the day of her transplant, her shunt was closed with a covered stent prior to her operation. From the St Louis experience, three children post-shunt came to transplant, two from progressive RV failure and one for hemoptysis (personal observation). At the time of transplant, two were on relatively high doses of IV prostacyclins and one was on veno-venous ECMO. All three transplants were marked by severe bleeding which resulted in two of the children dying post-operatively. The one survivor recovered fully. Reasons for the profound bleeding are likely multifold and include the known risk that prior thoracotomies have on chest wall neo-vascularization and lung adhesions, failure to adequately control the shunt at the time of transplant, and clotting derangements due to medications such as prostacyclins. One technical modification that might reduce these complications is to place a shunt through a sternotomy. This approach could potentially reduce lung adhesions, provide better control of the shunt at the time of transplant, and eliminate the need for any additional peri-operative procedures. ${ }^{24}$ Overall, while having a Potts shunt does not prevent future consideration of lung transplantation, it does appear to increase the risk and technical challenge of the operation. Lung transplantation in of itself is a complex procedure. Consequently, performing one on a child who has had a Potts shunt is not something every transplant center is willing to consider. Therefore, before proceeding with a Potts shunt, frank discussions with families about possible future limitations and risks would obviously be important in helping them make informed decisions.

\section{Conflict of Interest}

The author declares no conflict of interest.

\section{References}

1. Barst RJ, Maislin G, Fishman AP. Vasodilator therapy for primary pulmonary hypertension in children. Circulation. 1999;99:1197-208.

2. Haworth SG, Hislop AA. Treatment and survival in children with pulmonary arterial hypertension: the UK Pulmonary Hypertension Service for Children 2001-2006. Heart. 2009;95:312-17.

3. Zijlstra WMH, Douwes JM, Rosenzweig EB, et al. Survival differences in pediatric pulmonary arterial hypertension. J Am Coll Cardiol. 2014;63:2159-69.

4. Goldfarb SB, Levvey BJ, Edwards LB, et al. The registry of the international society for heart and lung transplantation: Nineteenth pediatric lung and heart-lung transplantation report-2016; focus theme: Primary diagnostic indications for transplant. J Heart Lung Transplant. 2016;35:1196-1205.

5. Goldstein BS, Sweet SC, Mao J, et al. Lung transplantation in children with idiopathic pulmonary arterial hypertension: An 18-year experience. J Heart Lung Transplant. 2011;30:1148-52.

6. Rozkovec A, Montanes P, Oakley CM. Factors that influence the outcome of primary pulmonary hypertension. Br Heart J. 1986;55:449-58.

7. Glanville AR, Burke CM, Theodore J, et al. Primary pulmonary hypertension. Length of survival in patients referred for heart-lung transplantation. Chest. 1987;91:675-81.

8. Bhamra-Ariza P, Keogh AM, Muller DWM. Percutaneous interventional therapies for the treatment of patients with severe pulmonary hypertension. J Am Coll Cardiol. 2014;63:611-8.

9. Sandoval J, Gomez-Arroyo J, Gaspar J, et al. Interventional and surgical therapeutic strategies for pulmonary arterial hypertension: Beyond palliative treatments. J Cardiol. 2015;66:304-14.

10. Micheletti A, Hislop AA, Lammers A, et al. Role of atrial septostomy in the treatment of children with pulmonary arterial hypertension. Heart. 2006;92:969-72.

11. Abman SH, Hansmann G, Archer SL, et al. Pediatric pulmonary hypertension. Guidelines form the American heart association and American thoracic society. Circulation. 2015;132:2037-99.

12. Chiu JS, Zuckerman WA, Turner ME, et al. Balloon atrial septostomy in pulmonary arterial hypertension: Effect on survival and associated outcomes. J Heart Lung Transplant. 2015;34:376-80.

13. Bauer A, Khalil M, Schmidt D, et al. Creation of a restrictive atrial communication in pulmonary arterial hypertension $(\mathrm{PAH})$ : effective palliation of syncope and end-stage heart failure. Pulmonary Circulation. 2017;8:1-9.

14. Potts WJ, Smith S, Gibson S. Anastomosis of the aorta to a pulmonary artery: certain types in 
congenital heart disease. J Am Med Assoc. 1946;132:627-31.

15. Blanc J, Vouhe P, Bonnet D. Pott shunt in patients with pulmonary hypertension. N Engl J Med. 2004;350:6.

16. Hopkins WE, Ochoa LL, Richardson GW, et al. Comparison of the hemodynamics and survival of adults with severe primary pulmonary hypertension or Eisenmenger syndrome. J Heart Lung Transplant. 1996;15:100-5.

17. Diller GP, Dimopoulos K, Broberg CS, et al. Presentation, survival prospects, and predictors of death in Eisenmenger syndrome: a combined retrospective and case-controlled study. Eur Heart J. 2006;27:173742.

18. Baruteau AE, Belli E, Boudjemline Y, et al. Eur J Cardio-Thorac Surg. 2014;1-6.

19. Grady RM, Eghtesady P. Potts shunt and pediatric pulmonary hypertension: What we have learned. Ann Thorac Surg. 2015;101:1539-43.

20. Barst RJ, Ivy DD, Foreman AJ, et al. Four- and seven-year outcomes of patients with congenital heart disease-associated pulmonary arterial hypertension (from the REVEAL registry). Am J Cardiol. 2014;113:147-55.

21. Ploegstra MJ, Douwes JM, Roofthooft MTR, et al. Identification of treatment goals in paediatric pulmonary arterial hypertension. Eur Resp J. 2014;44:1616-26.

22. Esch JJ, Shah PB, Cockrill BA, et al. Transcatheter Potts shunt creation in patients with severe arterial hypertension: initial clinical experience. J Heart Lung Transplant. 2013;32:381-7.

23. Boudjemline Y, Sizarov A, Malekzadeh-Milani S, et al. Safety and feasibility of the transcatheter approach to create a reverse Potts shunt in children with idiopathic pulmonary arterial hypertension. Can J Cardiol. 2017;33:1188-96.

24. Rosenzweig EB, Ankola A, Krishnan U, et al. A novel unidirectional-valved shunt approach for endstage pulmonary arterial hypertension: Early experience in adolescents and adults. J Thorac Cardiovasc Surg. 2019; online ahead of print.

25. Paradela M, Mercier O, Baruteau A, et al. Endovascular closure of Potts shunt before double lung transplantation for idiopathic pulmonary arterial hypertension. J Thorac Cardiovasc Surg. 2013;146:e57. 